\title{
Retrograde transradial Approach for Hemodialysis Access Intervention: A Single-Center Study
}

\author{
G.M. Mokbul Hossain ${ }^{1}$, Naresh Chandra Mandal ${ }^{2}$, Rakibul Hasan ${ }^{3}$, Nirmal Kanti Dey ${ }^{4}$ Abdullah Al-Mamun ${ }^{5}$, \\ SMG Saklayen ${ }^{6}$, Swadesh Ranjan Sarker ${ }^{7}$, Motiur Rahman Sarker $^{8}$, AKM Ziaul Huque ${ }^{9}$, Shajadi Ferdous ${ }^{10}$, \\ Md. Mujibur Rahman Rony ${ }^{11}$
}

\begin{abstract}
:
Perianastomotic stenosis is a common scenario after creation of arteriovenous fistula for hemodialysis. Most of the interventionists prefer transvenous approach. But transradial approach can easily visualize radial artery and cephalic venous tree up to central vein. This retrospective study was performed from November 2012 to January 2017 in Ibn Sina Hospital, Dhanmondi, Dhaka. Total patients undergoing hemodialysis access were 148 (male 74, female 74, male-female ratio 1:1). Number of radiocephalic fistula was 95 (64\%), brachiocephalic fistula 50 (34\%) \& others $3(2 \%)$. Most of the punctures were done by palpation. Sometimes puncture was made by
\end{abstract}

ultrasonogram guidance. Puncture needle size was 21 gauge, $2.5 \mathrm{~cm}$ or $4 \mathrm{~cm}$ long. Sheath size was $6 \mathrm{~F} \mathrm{x} 4 \mathrm{~cm}$ or $7 \mathrm{~F} \times 4 \mathrm{~cm}$. Majority of the cases (140) were successfully approached through retrograde transradial route. Few cases (8) were approached through retrograde venous route due to thrombosis of radial artery for previous intervention or creation of radiocephalic fistula in an end to end fashion. It was concluded that retrograde transradial approach to dilate perianastomotic stenosis as well as outflow vein is a good option.

Key words: Arteriovenous Fistula (AVF), Transradial Approach (TRA)
Introduction:

Maintenance of dialysis access patency is crucial for patients with end-stage renal disease who are on hemodialysis. Dysfunctional dialysis access is a

1. Associate Professor, Vascular Surgery, NICVD, Dhaka.

2. Professor, Vascular Surgery, NICVD, Dhaka

3. Assistant Professor, Vascular Surgery, BSMMU, Dhaka

4. Assistant Professor, Vascular Surgery, NICVD, Dhaka

5. Assistant Professor, Vascular Surgery, NICVD, Dhaka

6. Assistant Professor, Vascular Surgery, Ibrahim Cardiac Hospital, Dhaka

7. Assistant Professor, Vascular Surgery, NICVD, Dhaka

8. Assistant Professor, Vascular Surgery, Chittagong Medical College, Dhaka

9. Assistant Registrar, Vascular Surgery, NICVD, Dhaka

10. Sonologist, Bangladesh Medical College Hospital, Dhaka

11. Assistant Registrar, Vascular Surgery, NICVD, Dhaka.

Address of Correspondence: Dr. G.M. Mokbul Hossain, Ibn Sina Hospital, House\#68, Road\#15/A, Dhanmondi, Dhaka, Bangladesh. E-mail: dr.mokbul@yahoo.com, Mobile: +8801819282857 common cause of morbidity and hospitalization in these patients. Stenotic lesions often threaten the patency of an arteriovenous (AV) access. These lesions can occur at various locations of the access circuit, including the juxta-anastomotic area, outûow vein, or central venous system. Percutaneous angioplasty is indicated in ûstulas with $>50 \%$ stenosis in arterial or venous limbs. ${ }^{1}$ The traditional approach for intervening on malfunctioning dialysis access has been the direct antegrade or retrograde puncture of the fistula itself.

Transvenous approach is generally considered the standard approach. Transvenous access is usually relatively simple because the access vein is dilated in mature fistulas and only rarely spasm. The dilated vein is also well suited to accommodate large sheaths that may be needed for larger diameter angioplasty balloons and stents. Drawbacks of the antegrade transvenous approach include 1) difficulty to identify the AV anastomosis and afferent artery during reflux angiography

DOI: https://doi.org/10.3329/bhj.v35i1.49143

Copyright $\odot 2017$ Bangladesh Cardiac Society. Published by Bangladesh Cardiac Society. This is an Open Access articles published under the Creative Commons Attribution-NonCommercial 4.0 International License (CC BY-NC). This license permits use, distribution and reproduction in any medium, provided the original work is properly cited and is not used for commercial purposes. 
that is done during cuff inflation or balloon occlusion of the venous outflow 2) a possible puncture site occlusion during compression after the procedure and 3 ) a potential risk for distal embolization into the hand when crossing anastomotic occlusive lesions containing thrombus in a retrograde fashion. The TRA approach offers unique potential advantages for the evaluation and treatment of dysfunctional AVFs. The radial artery is easily accessible by manual palpation or duplex sonography, and operator fluoroscopic exposure is substantially reduced with this approach. Most fundamentally, the radial approach permits evaluation and treatment of the entire fistula from the afferent artery through to the central veins with only a single puncture. Finally, postprocedure hemostasis of a radial puncture is easily achieved despite the intraprocedural use of heparin or thrombolytic agents because of its favorable anatomic location and easy compressibility. There are also several potential drawbacks to the use of TRA. Technically, the TRA puncture can be difficult for those who are not comfortable with this technique; however, there is short learning curve to perform the puncture. Puncture of the radial artery may be difficult if the pulse is weak. This can be overcome with the use of ultrasonography.

Access site for diagnosis and management of any hemodialysis fistula varies due to operator preferences, suspected type and sites of abnormality and the anatomic pattern of the fistula. Decisions regarding the puncture sites are predicated on physical examination, knowledge of previous surgery and review of previous imaging studies. The goal for the interventionist is to choose an access site that is well suited to identify and treat all of these lesions to reduce needle punctures, lessen procedure time and variation, and allow rapid restoration of access function so that the patient can quickly return to hemodialysis. Figure 1 shows different types of arteriovenous fistula for hemodialysis.

\section{Material and Methods:}

We retrospectively studied 148 cases from 2012 to 2017. Inclusion criteria included palpable radial artery \& dysfunctional radiocephalic or brachiocephalic or brachiobasilic transposition fistula. Exclusion criteria included impalpable radial artery, oximetry $<90 \%$ after occlusion of radial artery, abnormal Allen's test or infected fistula. Number of successful puncture of radial artery followed by fistuloplasty was evaluated. Periprocedural complications like radial artery thrombosis dissection or hematoma were also evaluated.

Technique of retrograde cannulation of radial artery: When considering a patient for TRA, the interventionist must evaluate the radial artery and assess the radial/ ulnar artery circulation. This can be accomplished by performing the Allen's test. To perform the Allen's test, both the radial and ulnar arteries are compressed until blanching of the hand occurs. The examiner then releases pressure on the ulnar artery to determine if flow returns to the radial aspect of the hand, implying patency

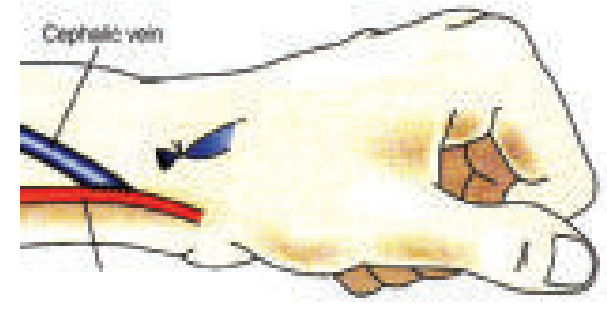

A) Radio-Cephalic Finteb

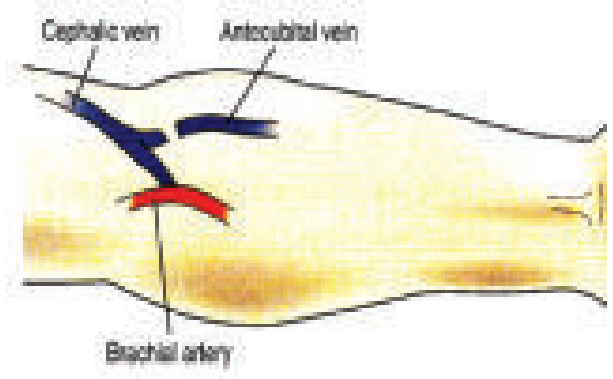

B) Brachio-Cephafis Fiula

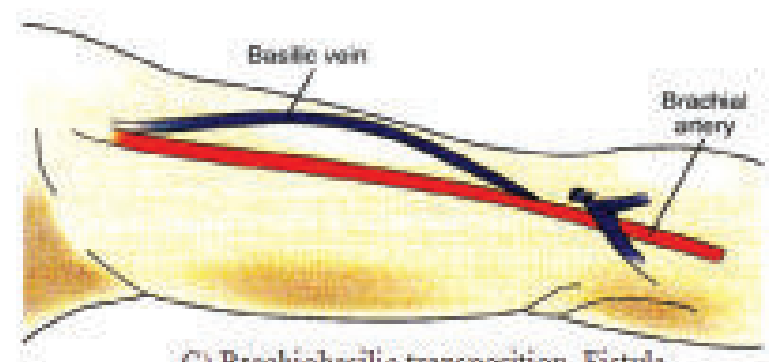

C) Brachiobisilis transposition Fistull

Fig.-1: Different types of arteriovenous fistula for hemodialysis 
of the palmar arch. If hyperemia does not occur (usually within 5-7 seconds), radial access should be avoided as the patient does not have dual circulation to the hand. TRA in cases of an abnormal Allen's test may result in hand ischemia and a substantial risk of radial artery occlusion. ${ }^{2}$ Correct positioning of the wrist is important. The wrist should be hyperextended $60^{\circ}$ and may be supported by placement of a towel underneath the wrist. Using physical examination or sonography, the radial artery should be punctured at least 2 to $3 \mathrm{~cm}$ distal to the arteriovenous anastomosis to allow sufficient distance for sheath insertion and anastomotic interventions. When evaluating upper arm fistulas using a TRA approach, the radial artery can be punctured at its most accessible location as determined by the pulse examination.

We generally perform the transradial puncture with a 21 gauge, 2.5 or $4 \mathrm{~cm}$ long needle either by direct palpation or under direct ultrasound guidance. Advancement of the 0.018 inch guidewire into the radial artery can be visualized with fluoroscopic guidance. In most cases, complete angiographic assessment of the dysfunctional AVF can be performed through $6 \mathrm{~F} \times 4 \mathrm{~cm}$ or $7 \mathrm{~F} \times 4 \mathrm{~cm}$ radial sheath. Interestingly, due to preferential flow into a nonthrombosed fistula, the direction of flow in the radial artery is often retrograde. Interestingly $60 \%$ volume of blood in venous limb composed from radial artery proximal to anastomosis and that of $40 \%$ from distal to anastomosis and the tip of the sheath are kept distal to anastomosis.

Using this approach, dilation and insertion of a larger sheath was only performed once it is deemed that intervention is required. We generally advanced a $6 \mathrm{~F}$ to $7 \mathrm{~F}$ sheath into the radial artery distal to the anastomosis. 2500 IU Heparin with 100 microgram GTN was injected via sheath and just before angioplasty another $2500 \mathrm{IU}$ heparin was injected via intravenous cannula. Once the anastomosis was seen, roadmap guidance was performed to guide catheterization of the fistula using a $4 \mathrm{~F}$ angled catheter. If it was necessary to maintain the wire position while injecting contrast, this can be achieved by injecting through the $4 \mathrm{~F}$ catheter over a 0.018 or 0.014 inch wire using an attached Check-Flo sidearm adapter (Cook Medical). It was performed in radial artery \& venous outflow upto central vein were easily visualized.

Figure 2 shows retrograde transradial approach, Figure 3 shows retgrograde transvenous approach to treat

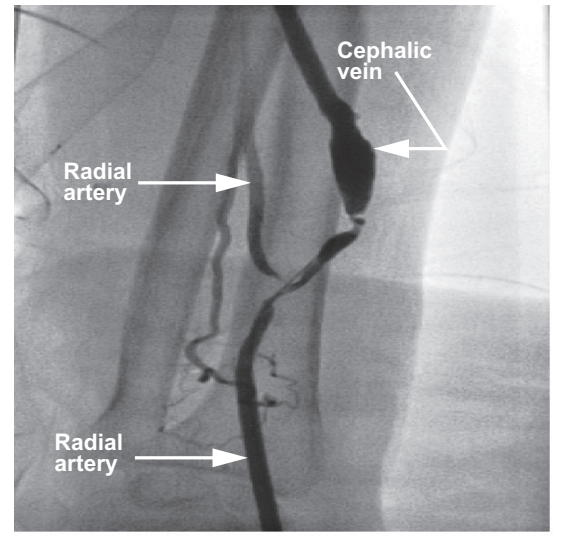

A: Preprocedure angiogram

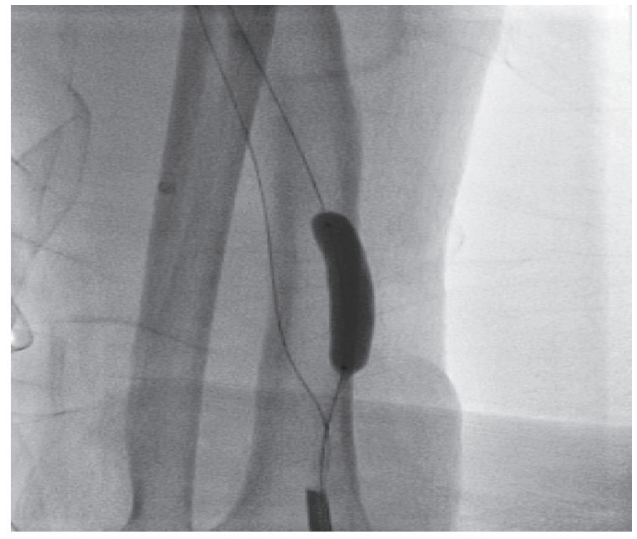

B: Balloon angioplasty

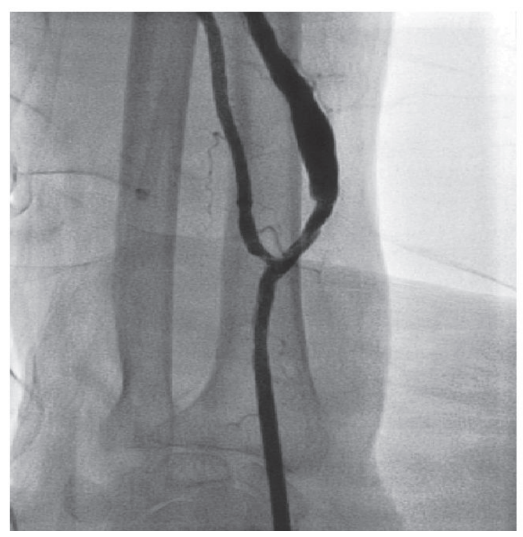

C: Post procedure angiogram

Fig.-2 (A, B, C): Retrograde transradial approach to treat radiocephalic fistula malfunction. 


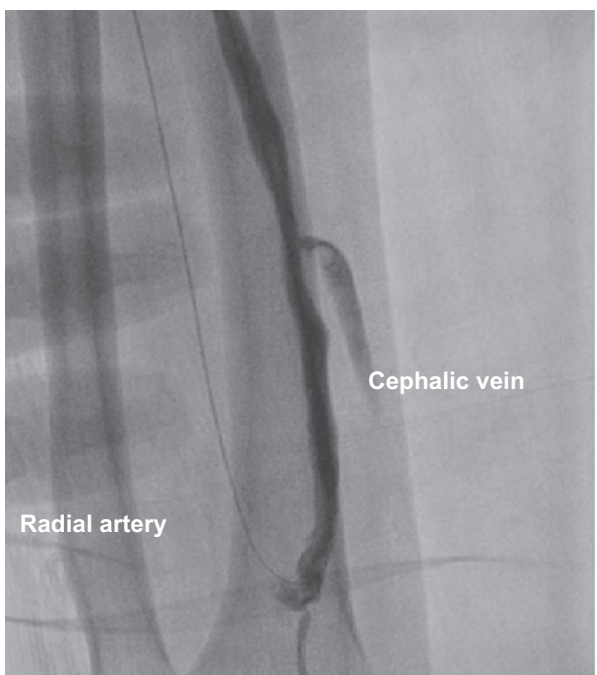

A: Preprocedure angiogram

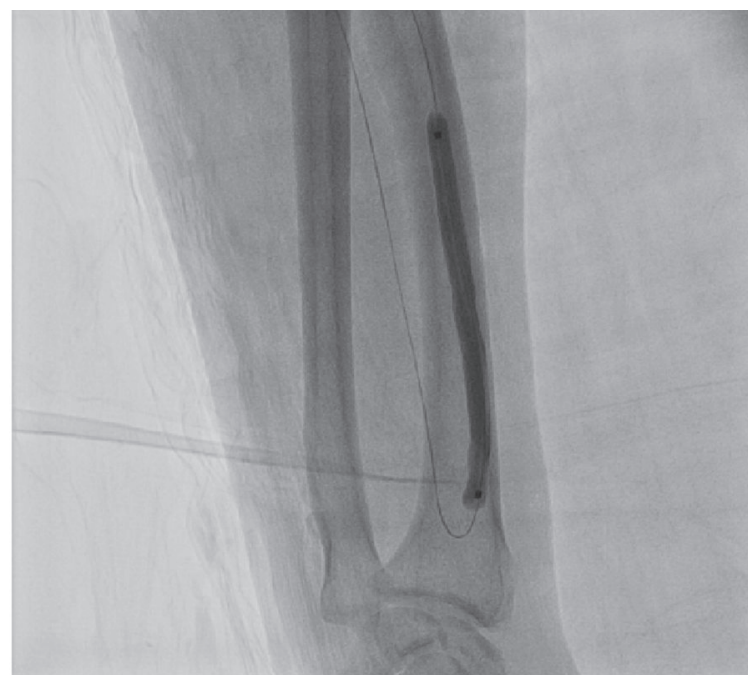

B: Balloon angioplasty

Fig.-3: Retrograde transvenous angioplasty of radiocephalic fistula

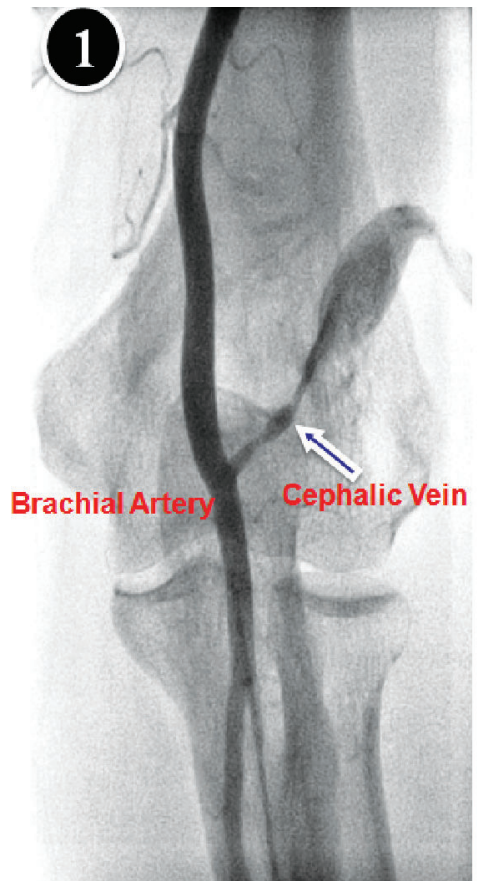

A: Preprocedure angiogram

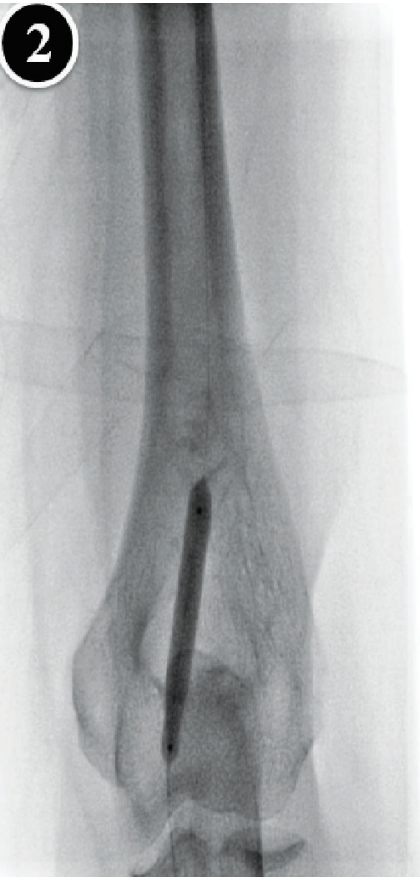

B: Balloon angioplasty

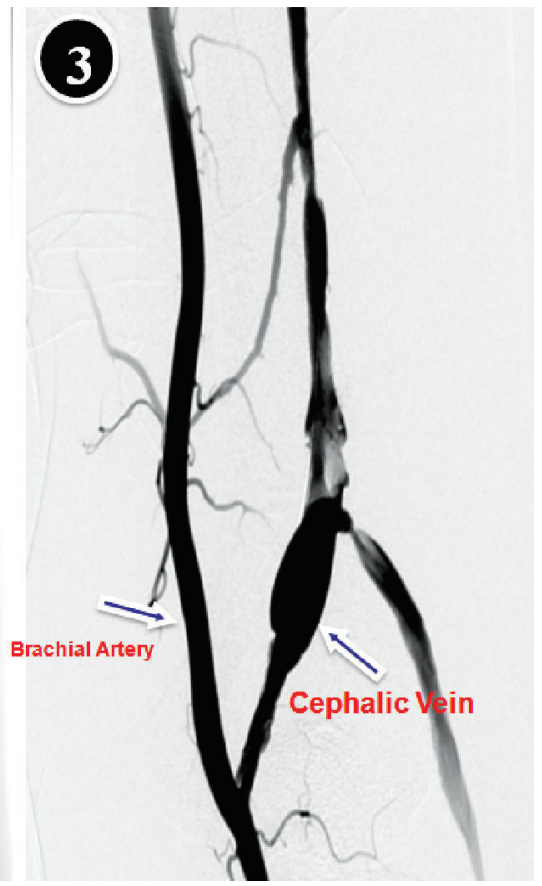

C: Post procedure angiogram

Fig.-4: Retrograde transradial approach for brachiocephalic fistula

radiocephalic fistula malfunction \& Figure 4 shows retrograde transradial approach to treat brachiocephalic fistula malfunction.

Once TRA was established, it was relatively simple to complete, including balloon angioplasty using standard noncompliant balloon. Dilation and mobilization of thrombus from the arterial anastomosis through the venous outflow was then performed using a 4- to 5-mm PTA balloon with low-pressure hand inflation. Once thrombus was cleared and flow was restored, angioplasty and other interventions were then performed in the usual fashion. One important technical 
consideration is notable in our experience. After the procedure, we did not use the radial artery compression bands that are typically described for hemostasis after transradial puncture for cardiac catheterization. Instead, we achieved hemostasis using nonocclusive manual compression. Despite this technical factor, we had no occurrence of hand ischemia and only rare instances in which there was subsequent occlusion of the radial artery preventing future access.

\section{Results:}

From November 2012 to January 2017, total 148 patients (male:female=1:1) were evaluated. Types of arteriovenous fistula were radiocephalic 95 (64\%), brachiocephalic 50 (34\%) \& brachiobasilic transposition 3 (2\%). 146 cases were approached through retrograde transradial artery \& only 2 cases through retrograde cephalic vein as they have side to side anastomosis. During secondary procedure 6 patients (4.1\%) developed radial artery thrombosis due to previous procedure and no patient developed hand ischemia or hematoma.

\section{Discussion:}

Now a day percutaneous fistuloplasty for hemodialysis is routinely done to maintain patency but most interventionist do it by retrograde venous approach. But transradial approach is an alternative to retrograde venous approach in patients having patent radial artery, intact palmar arch, juxta anastomotic stenosis, multiple venous outflow stenoses or lesions in both arterial \& venous limbs. ${ }^{3}$

By using one retrograde radial sheath, one can evaluate both arterial side as well as venous limb upto central vein. Transradial approach can solve juxta-anastomic lesion as well as lesions in outflow vein.

The use of transradial approach (TRA) is a widely accepted and well-described technique for coronary artery interventions and for the diagnosis and treatment of iliac and mesenteric diseases. ${ }^{4}$ Since 2006, several reports from Asian investigators have shown that the use of TRA for the dysfunctional AVF is safe and feasible. ${ }^{5-8}$ Kawarada et al initially described the use of TRA in 11 patients with predominantly nonthrombosed BresciaCimino fistulas. There was no incidence of hand ischemia or puncture site complications observed out to 6 months after intervention. ${ }^{9}$ Heparin and isosorbide dinitrate were administered through the radial sheath to prevent spasm and thrombosis. ${ }^{5}$ Lin et al subsequently reported on transradial interventions in 165 upper arm fistulas, more than half of which were performed for thrombosed accesses. Heparin was used, but vasodilator was not administered. Two episodes of radial artery spasm causing thrombosis and two asymptomatic radial dissections occurred, although no patient developed hand ischemia. ${ }^{8}$ Chen and associates described their experience with 154 transradial BresciaCimino fistula treatments (including 99 thrombosed accesses). There was a differential technical success for nonoccluded fistulas compared to fistulas having a "fibrous" occlusion, with success noted in $99 \%$ and $46 \%$, respectively. Overall 30-day primary patency was $75 \%{ }^{5}$ Taken together, the anatomic or clinical success rates from these reports are comparable to those reported in previous studies using the more traditional transvenous approach. ${ }^{5-8}$

The TRA approach is not recommended in patients with a positive Allen test result, and caution should be exercised in using this approach in patients without a palpable radial pulse. Second, this approach can be associated with a learning curve, and it is key that the operator be proûcient at using ultrasound imaging to gain anterior wall access by a single puncture only. Multiple failed attempts may lead to radial artery spasm and hematoma. Third, a hydrophilic transradial tapered sheath allows for a smooth entry into the radial artery, thereby decreasing the chance of a radial artery injury or rupture. Lastly, use of a solution containing a vasodilator and a calcium channel blocker intermittently during the TRA intervention minimizes radial artery vasospasm and thrombosis.

Infiltration of the subcutaneous periradial tissues with lidocaine containing $500 \mu \mathrm{g}$ of nitroglycerin can also cause radial artery vasodilation and facilitate puncture. ${ }^{10}$ Temporary radial artery occlusion can occur during puncture and was seen in $<5 \%$ of patients undergoing TRA for coronary interventions. ${ }^{11}$ Repeated puncture of the radial artery for access may lead to eventual occlusion or stenosis and prevent this approach for access; however, Chen et al found that $98.7 \%$ (152/154) of patients had a good palpable pulse at follow-up. ${ }^{6}$ Due to the relatively small size of the radial artery, sheath size may be limited to a $6-\mathrm{F}$ or $7-\mathrm{F}$ sheath. This potentially prevents the use of larger balloon catheters for central venous angioplasty, necessitating an additional transvenous puncture if such devices are necessary. The availability of newer low profile monorail balloons (e.g., Sterling balloon catheter, Boston Scientific Corporation, Natick, MA) provides the ability to perform $8 \mathrm{~mm}$ percutaneous transluminal angioplasty.

Multiple studies have also documented that the TRA for coronary interventions has lower bleeding and access site complications compared with brachial 
approaches. $3,14,15$ The complication rate for a brachial approach can be up to $12 \% ;^{16}$ by comparison, complication rates for radial artery approaches are very minimal. Puncture failure rates and radial artery occlusions are as low as $0.25 \%$ and $0.7 \%$ respectively. ${ }^{17,18}$

In a recent study, no local complications from the puncture site occurred and 6 patients underwent multiple TRA procedures with thrombosis of radial artery. No patients complained of hand ischemia or hematoma in followup. Patients seen in the clinic underwent a clinical pulse examination; therefore, an arterial duplex to evaluate radial artery patency at post intervention was not routinely ordered. ${ }^{19}$ In our study, complication rate was $4.1 \%$. Wu et al documented a complication rate of $4 \%$ in their study of the TRA approach in occluded radiocephalic AVFs. ${ }^{20}$ Lin et al reported a complication rate of $9.7 \%$ that included interventions for stenosis and thrombosis in AVFs. ${ }^{21}$

A limitation to the TRA is the inability to accommodate large sheath sizes, although most interventions in the AV access should be able to perform through a 6F sheath. In our study, the largest sheath size used for a TRA was 7F. Large noncompliant balloons that treat central venous lesions require larger sheaths, thereby making the TRA approach a poor option in these cases.

In our study, we highlighted the versatility of the TRA approach as evidenced by its efûcacy in treating juxtaanastomotic lesions as well as treating different AVF types. The most common type of AVF treated in this cohort was the distal radiocephalic conûguration. We have proven that despite minimal running room, distal radiocephalic AVFs can be treated successfully. Accessing the radial artery allows a more direct route to the lesion than the traditional venous route that may require crossing difûcult angles and kinks to reach a juxta-anastomotic lesion.

\section{Conclusion:}

Retrograde transradial approach is safe and effective to treat malfunctioning hemodialyhsis access. It allows visualization as well as treatment of stenosis or occlusion of arterial and venous limbs in a single puncture. The procedure is easy to learn having very few complications.

\section{References:}

1. Vascular Access. 2006 Work Group. Clinical practice guidelines for vascular access. Am J Kidney Dis. 2006 Jul;48(Suppl 1):176-247.

2. Greenwood MJ, Della-Siega AJ, Fretz EB, Kinloch $\mathrm{D}$, Klinke $\mathrm{P}$, Mildenberger $\mathrm{R}$, et al. Vascular communications of the hand in patients being considered for transradial coronary angiography: is the Allen's test accurate? J Am Coll Cardiol. 2005 Dec;46(11):2013-2017.

3. Clark TWI, Hirsch DA, Jindal KJ, Veugelers PJ, LeBlanc J. Outcome and prognostic factors of restenosis after percutaneous treatment of native hemodialysis fistulas. J Vasc Interv Radiol. 2002 Jan;13(1):51-59.

4. Linda L, Brooks A, Donovan M, Smith TA, Sternbergh III WC, Bazan HA. Transradial approach for percutaneous intervention of malfunctioning arteriovenous accesses. J Vasc Surg. 2015 Mar;61(3):747-753.

5. Kawarada O, Yokoi Y, Nakata S, Morioka N, Takemoto $\mathrm{K}$. Transradial intervention for native fistula failure. Catheter Cardiovasc Interv. 2006 Oct;68(4):513-520.

6. Chen SM, Hang CL, Yip HK, Fang CY, Wu CJ, Yang $\mathrm{CH}$, et al. Outcomes of interventions via a transradial approach for dysfunctional BresciaCimino fistulas. Cardiovasc Intervent Radiol. 2009 Sep;32(5):952-959.

7. Wu CC, Wen SC, Chen MK, et al. Radial artery approach for endovascular salvage of occluded autogenous radial-cephalic fistulae. Nephrol Dial Transplant. 2009;24(8):2497-2502.

8. Wang HJ, Yang YF. Percutaneous Treatment of Dysfunctional Brescia-Cimino Fistulae Through a Radial Arterial Approach. Am J Kidney Dis. 2006 Oct;48(4):652-658.

9. Basile A, Medina JG, Lupattelli T, Medina VG, Leal $R$. Internal jugular vein access for the interventional management of nonfunctioning artero-venous haemodialysis fistulas. Eur J Radiol. 2004 Dec;52(3):288-292.

10. Candemir B, Kumbasar D, Turhan S, Klickap M, Ozdol C, Akyurek O, et al. Facilitation of radial artery cannulation by periradial subcutaneous administration of nitroglycerin. J Vasc Interv Radiol. 2009 Sep;20(9):1151-1156.

11. Stella PR, Kiemeneij F, Laarman GJ, Oderkerken D, Slagboom T, Wieken RV. Incidence and outcome of radial artery occlusion following transradial artery coronary angioplasty. Cathet Cardiovasc Diag. 1998 Dec 06;40(2):156-158.

12. Rao SV, Cohen MG, Kandzari DE, Bertrand OF, Gilchrist IC. The transradial approach to 
percutaneous coronary intervention: historical perspective, current concepts, and future directions. J Am Coll Cardiol. 2010 May;55(20):2187-2195.

13. Doyle BJ, Rihal CS, Gastineau DA, Holmes DR. Bleeding, Blood Transfusion, and Increased Mortality After Percutaneous Coronary Intervention. Implications for Contemporary Practice. Journal of the American College of Cardiology. 2009 Jun;53(22):2019-2027.

14. Manninen HI, Kaukanen ET, Ikäheimo R, Karhapää P, Lahtinen T, Matsi P, et al. Brachial arterial access: Endovascular treatment of failing Brescia-Cimino hemodialysis fistulas - Initial success and longterm results. Radiology. 2001 Mar 01;218(3):711718.

15. Jeon UB, Kim CW, Chung SW. Percutaneous treatment of thrombosed prosthetic brachial-basilic access by the transradial approach. J Vasc Surg. 2009 Apr;49(4):1057-1059.

16. Wang S, Asif A. Transradial Approach for Cardiovascular Interventions and Its Implications for Hemodialysis Vascular Access. Seminars in Dialysis. 2012 Nov 22;26(3):E20-E29.
17. Beathard GA. Percutaneous transvenous angioplasty in the treatment of vascular access stenosis. Kidney Int. 1992 Dec;42(6):1390-1397.

18. Wu CC, Wen SC, Chen MK, Yang CW, Pu SY, Tsai $\mathrm{KC}$, et al. Radial artery approach for endovascular salvage of occluded autogenous radial-cephalic fistulae. Nephrol Dial Transplant. 2009 Aug;24(8):2497-2502.

19. Lin YS, Lin PC, Hsu J Te, Chang ST, Yang TY, Cheng HW, et al. Feasibility of trans-radial approach in percutaneous intervention for upper arm dialysis access. Semin Dial. 2008 Dec 1;21(6):567-574.

20. Campeau L. Percutaneous radial artery approach for coronary angiography. Cathet Cardiovasc Diagn 1989;16(1):3-7. Available from: https:// onlinelibrary.wiley.com DOI: 10.1002/ ccd.1810160103

21. Caputo RP, Tremmel JA, Rao S, Gilchrist IC, Pyne $\mathrm{C}$, Pancholy S, et al. Transradial arterial access for coronary and peripheral procedures: executive summary by the Transradial Committee of the SCAI. Catheter Cardiovasc Interv. 2011 Nov 15;78(6):823839. 